Research Article

\title{
Stability Analysis of Optimal Trajectory for Nonlinear Optimal Control Problems
}

\author{
Hongyong Deng $(\mathbb{D}$, Wei Zhang, and Changchun Shen \\ School of Data Science and Information Engineering, Guizhou Minzu University, Guiyang, Guizhou, China \\ Correspondence should be addressed to Hongyong Deng; dhycel@vip.163.com
}

Received 7 June 2020; Accepted 1 August 2020; Published 27 August 2020

Academic Editor: Basil K. Papadopoulos

Copyright ( $) 2020$ Hongyong Deng et al. This is an open access article distributed under the Creative Commons Attribution License, which permits unrestricted use, distribution, and reproduction in any medium, provided the original work is properly cited.

Due to the need for numerical calculation and mathematical modelling, this paper focuses on the stability of optimal trajectories for optimal control problems. The basic ideas and techniques are based on the compactness of the optimal trajectory set and setvalued mapping theorem. Through lack of optimal control stability, the result of generic stability for optimal trajectories is obtained under the perturbations of the right-hand side functions of the state equations; in the sense of Baire category, the righthand side functions of the state equations of optimal control can be approximated by other functions.

\section{Introduction}

Due to the need for numerical calculation and mathematical modelling, we will consider the influence of optimal trajectory with the changing right-hand side functions of the state equations on the approximate functions. There are many experts who have done much work on the stability of the optimal control problem [1-4], and especially in the case of the disturbance of the right-hand side function, the stability of the optimal control is discussed [5-7]. However, in the actual problem, the real decisive factor for the optimal control problem is the stability of the optimal trajectory, and sometimes, the optimal control does not necessarily converge; that is, when the trajectory converges, the corresponding control does not necessarily converge. Therefore, this paper discusses the stability in these cases, focusing on two problems: First, the compactness of the feasible trajectories corresponding to the perturbations of right-hand side functions of the state equations is discussed, and then, the compactness of the optimal trajectories is discussed. Second, the stability of the optimal trajectories set corresponding to the perturbations of the right-hand side functions is discussed.

Consider Bolza problem, Problem P, as follows:
Find an optimal pair $(\bar{y}(\cdot), \bar{u}(\cdot)) \in p_{a d}[0, T]$ such that the cost functional

$$
J(y(\cdot), u(\cdot))=h(y(T))+\int_{0}^{T} f^{0}(t, y(t)) \mathrm{d} t
$$

reaches its minimum at $(\bar{y}(\cdot), \bar{u}(\cdot))$ for all $(y(\cdot), u(\cdot)) \epsilon$ $P_{a d}[0, T]$, where the admissible pair $(y(\cdot), u(\cdot))$ are solutions of the following nonlinear controlled system:

$$
\left\{\begin{array}{l}
\dot{y}(t)=f(t, y(t), u(t)), t \in[0, T], \\
y(0)=y_{0} .
\end{array}\right.
$$

Here, $u(t) \in \mathscr{U}[0, T]$ and $P_{a d}[0, T]$ is the set of all admissible pair, and the set of admissible controls is defined by

$$
\mathcal{U}[0, T] \equiv\{u:[0, T] \longmapsto U \mid u(\cdot) \text { is measurable }\},
$$

where $U$ is a metric space.

In order to discuss the optimal control problem (P), let us start with some basic assumptions:

(H1) The terminal time $T>0$ is fixed, and the metric space $U$ is compact. 
(H2) The function $f:[0, T] \times R^{n} \times U \longmapsto R^{n}$ is measurable w.r.t. $t \in[0, T]$, continuous concerning $u \in U$. There exist $L, C>0$ such that

$\|f(t, x, u)-f(t, y, u)\| \leq L\|x-y\|$, $\|f(t, x, u)\| \leq C$, for all $x, y \in R^{n}, t \in[0, T], u \in U$.

(H3) The function $f:[0, T] \times R^{n} \times U \longmapsto R^{n}$ satisfies the following Filippov-Roxin condition:

The set $f(t, y, U)=\{f(t, y, u) \mid u \in U\}$ is convex and closed for almost all $t \in[0, T]$ and all $y \in R^{n}$

(H4) The function $f^{0}:[0, T] \times R^{n} \longmapsto R$ is Borel measurable concerning $t \in[0, T]$ and continuous concerning $y \in R^{n}$, and $h: R^{n} \longmapsto R$ is continuous.

Under the assumptions and from [8], equation (2) has a unique solution. Also, the solution of equation (2) depends continuously on the right-hand side function and is differentiable concerning initial data.

This paper is organized as follows. In part one, we construct the complete metric space of $f$ satisfied the conditions of $(\mathrm{H} 2-\mathrm{H} 3)$ and discuss the compactness of the feasible trajectories set. In part two, we consider the stability of optimal trajectory in sense of Baire category. In the last section, we give an example and conclusion.

\section{Compactness of Optimal Trajectory}

For the aim to consider the stability of optimal trajectory, first, we need some notions.

Let

$Y=\{f \mid f$ satisfies the conditions of (H2) and (H3) $\}$.

For each $f_{1}, f_{2} \in Y$, define

$$
\rho\left(f_{1}, f_{2}\right)=\sup _{(t, y, u) \in[0, T] \times R^{n} \times U}\left\|f_{1}(t, y, u)-f_{2}(t, y, u)\right\|,
$$

then we know that space $(Y, \rho)$ is a complete metric space easily.

Set

$$
\begin{aligned}
S(f)= & \{\bar{y} \mid \bar{y} \text { are optimal trajectories of optimal control problems } \\
& \cdot(P) \text { for each } f \in Y\} .
\end{aligned}
$$

Then, the correspondence $f \longmapsto S(f)$ is a set-valued mapping; for convenience to discuss with the next, we expand the scope of $S(f)$ into $\mathscr{Y}_{S}[0, T] \subseteq C\left([0, T] ; R^{n}\right)$, where $\mathscr{Y}_{S}[0, T] \subseteq C\left([0, T] ; R^{n}\right)$ is the set of all feasible trajectories of optimal control problems (P) for each $f \in Y$, and we denote the set-valued mapping by $S: Y \longmapsto 2^{\mathscr{Y}_{S}[0, T]}$.

Now, we consider the properties of feasible trajectories set $\mathscr{Y}_{S}[0, T] \subseteq C\left([0, T] ; R^{n}\right)$; we have the following theorem.
Theorem 1. Suppose assumptions (H1) and (H2) hold, then the feasible trajectories set $\mathscr{Y}_{S}[0, T]$ is relatively compact in $C\left([0, T] ; R^{n}\right)$

Proof. By the assumptions of ( $\mathrm{H} 1)$ and $(\mathrm{H} 2)$, equation (2) has a unique solution for each $f \in Y$, and the solution is denoted by $y_{f}(t, u(\cdot))=y_{0}+\int_{0}^{t} f(\tau, y(\tau), u(\tau)) \mathrm{d} \tau$. Thanks to the assumption (H2), it follows that

$$
\begin{aligned}
\left\|y_{f}\right\| & =\left\|y_{0}+\int_{0}^{t} f(\tau, y(\tau), u(\tau)) \mathrm{d} \tau\right\| \\
& \leq\left\|y_{0}\right\|+\int_{0}^{t}\|f(\tau, y(\tau), u(\tau))\| \mathrm{d} \tau \\
& \leq\left\|y_{0}\right\|+\int_{0}^{t} C \mathrm{~d} \tau \\
& \leq\left\|y_{0}\right\|+\int_{0}^{T} C \mathrm{~d} \tau .
\end{aligned}
$$

Namely,

$\left\|y_{f}(t, u(\cdot))\right\| \leq C T+\left\|y_{0}\right\|$, for each $f \in Y, u(\cdot) \in \mathcal{U}[0, T]$.

This means that the feasible trajectories set $\mathscr{Y}_{S}[0, T]$ is uniformly bounded.

Furthermore, we obtain

$$
\begin{aligned}
\left\|y_{f}(t, u(\cdot))-y_{f}(s, u(\cdot))\right\| & \leq\left|\int_{s}^{t}\|f(\tau, y(\tau), u(\tau))\| \mathrm{d} \tau\right| \\
& \leq\left|\int_{s}^{t} C \mathrm{~d} \tau\right| .
\end{aligned}
$$

Hence,

$\left\|y_{f}(t, u(\cdot))-y_{f}(s, u(\cdot))\right\| \leq C|t-s|$, for all $s, t \in[0, T]$.

That is, the feasible trajectories set $\mathscr{Y}_{S}[0, T]$ is equicontinuous.

From (7)-(11), by the Arzela-Ascoli theorem, we prove this theorem.

Theorem 2. If assumptions (H1), (H2), (H3), and (H4) are satisfied, the feasible trajectories set $\mathcal{Y}_{S}[0, T]$ is compact in $C\left([0, T] ; R^{n}\right)$.

Proof. Let $\left\{y_{k}(\cdot)\right\} \subseteq \mathscr{Y}_{S}[0, T]$ and $y_{k}(\cdot)$ be the solution of the following equations:

$$
\left\{\begin{array}{l}
\dot{y}_{k}(t)=f_{k}\left(t, y_{k}(t), u_{k}(t)\right), \quad t \in[0, T], \\
y_{k}(0)=y_{0}
\end{array}\right.
$$

under the assumptions $(\mathrm{H} 1)-(\mathrm{H} 4)$ and $f_{k} \in Y$. Let $y_{k}(\cdot)=$ $y_{f_{k}}\left(\cdot, y_{0}, u_{k}(\cdot)\right)$ and assume that 


$$
y_{k}(\cdot) \longrightarrow \bar{y}(\cdot) \text { in } C\left([0, T] ; R^{n}\right) .
$$

Next, we need to show that $\bar{y}(\cdot) \in \mathscr{Y}_{S}[0, T]$.

Assume that

$$
f_{k}\left(\cdot, y_{k}(\cdot), u_{k}(\cdot)\right) \stackrel{w}{\longrightarrow} \bar{f}(\cdot) \text { in } L^{p}\left([0, T] ; R^{n}\right),
$$

where $p>1$, and we have $\bar{f} \in Y$. By the Mazur theorem, there exist convex combinations with $\alpha_{i}^{k} \geq 0, \sum_{i \geq 1} \alpha_{i}^{k}=1$, such that

$$
\sum_{i \geq 1} \alpha_{i}^{k} f_{k+i}\left(\cdot, y_{k+i}(\cdot), u_{k+i}(\cdot)\right) \longrightarrow \bar{f}(\cdot) \text { in } L^{p}\left([0, T] ; R^{n}\right) .
$$

By (H2) and (13), we have

$$
\begin{aligned}
& \left|\sum_{i \geq 1} \alpha_{i}^{k} \bar{f}\left(t, y_{k+i}(t), u_{k+i}(t)\right)-\sum_{i \geq 1} \alpha_{i}^{k} \bar{f}\left(t, \bar{y}(t), u_{k+i}(t)\right)\right| \\
& \leq L \sum_{i \geq 1} \alpha_{i}^{k}\left|y_{k+i}(t)-\bar{y}(t)\right| \longrightarrow 0(k \longrightarrow+\infty), \\
\bar{y}(t) & =\lim _{k \longrightarrow \infty} y_{k}(t) \\
& =\lim _{k \longrightarrow \infty}\left[y_{0}+\int_{0}^{t} f_{k}\left(s, y_{k}(s), u_{k}(s)\right) \mathrm{d} s\right] \\
& =y_{0}+\int_{0}^{t} \bar{f}(s) \mathrm{d} s \\
& =y_{0}+\int_{0}^{t} \lim _{k \longrightarrow \infty} \sum_{i \geq 1} \alpha_{i}^{k} f_{k+i}\left(s, y_{k+i}(s), u_{k+i}(s)\right) \mathrm{d} s \\
& =y_{0}+\int_{0}^{t} \lim _{k \longrightarrow \infty} \sum_{i \geq 1} \alpha_{i}^{k} \bar{f}\left(s, y_{k+i}(s), u_{k+i}(s)\right) \mathrm{d} s \\
& =y_{0}+\int_{0}^{t} \lim _{k \longrightarrow \infty} \sum_{i \geq 1} \alpha_{i}^{k} \bar{f}\left(s, \bar{y}(s), u_{k+i}(s)\right) \mathrm{d} s .
\end{aligned}
$$

Consider

$$
\begin{aligned}
\bar{f}(t) & =\lim _{k \longrightarrow \infty} f_{k} \\
& =\lim _{k \longrightarrow \infty} \sum_{i \geq 1} \alpha_{i}^{k} \bar{f}\left(t, \bar{y}(t), u_{k+i}(t)\right) \\
\epsilon \overline{c o} \bar{f}(t, \bar{y}(t), U) & =\bar{f}(t, \bar{y}(t), U) .
\end{aligned}
$$

By the Filippov's Lemma, there exists $\bar{u} \in \mathscr{U}[0, T]$ such that (17) is satisfied, namely,

$$
\bar{y}=y_{0}+\int_{0}^{t} \bar{f}(s, \bar{y}(s), \bar{u}(s))
$$

holds.

\section{Stability of Optimal Trajectory}

In this section, we discuss the stability of optimal trajectory based Baire category.

Theorem 3. Let (H1)-(H4) hold and $S(f) \neq \varnothing$ for each $f \in Y$.
Proof. For any fixed $f \in Y$, let $\left(y_{k}(\cdot), u_{k}(\cdot)\right) \in P_{a d}[0, T]$, and we have $y_{k}(\cdot) \in \mathscr{Y}_{S}[0, T]$; by the compactness of $\mathscr{Y}_{S}[0, T]$, there exists $\bar{y}(\cdot)=y\left(\cdot, y_{0}, \bar{u}(\cdot)\right) \in \mathscr{Y}_{S}[0, T]$ and $\bar{u}(\cdot) \in \mathscr{U}[0, T]$, such that $y_{k}(\cdot) \longmapsto \bar{y}(\cdot)$.

By assumption of (H4), we have

$$
\left\{\begin{array}{l}
f^{0}(t, \bar{y}(t))=\lim _{k \longrightarrow \infty} f^{0}\left(t, y_{k}(t)\right), \\
h(\bar{y}(T))=\lim _{k \longrightarrow \infty} h\left(y_{k}(T)\right) .
\end{array}\right.
$$

Namely, $J_{f}\left(y_{k}(\cdot)\right) \longrightarrow J_{f}(\bar{y}(\cdot))$, so $J_{f}(\cdot)$ is continuous concerning $y \in \mathscr{Y}_{S}[0, T]$; since $\mathscr{Y}_{S}[0, T]$ is a compact set, the minimum value of $J_{f}(\cdot)$ exists. Then, $S(f) \neq \varnothing$ for each $f \in Y$.

Theorem 4. Let (H1)-(H4) hold, and $S(f)$ is closed for each $f \in Y$.

Proof. By the compactness of $\mathscr{Y}_{S}[0, T]$, next we show that $\operatorname{Graph}(S)=\left\{(f, y) \in Y \times \mathscr{Y}_{S}[0, T] \mid y \in S(f)\right\}$ is closed. Take $\left\{f_{k}\right\} \subset Y$ with $f_{k} \longrightarrow f \in Y$ and $\bar{y}_{k} \in S\left(f_{k}\right)$ with $\bar{y}_{k} \longrightarrow \bar{y} \in \mathscr{Y}_{S}[0, T]$; first, we need to show $\bar{y} \in S(f)$.

Due to $\bar{y}_{k} \in S\left(f_{k}\right)$, for all $u \in \mathscr{U}[0, T]$, we have

$$
J_{f_{k}}\left(\bar{y}_{k}\right) \leq J_{f_{k}}\left(y_{k}\right) \text {, }
$$

and by (H4), we see that

$$
J_{f_{k}}\left(\bar{y}_{k}\right) \longrightarrow J_{f}(\bar{y}) .
$$

For any fixed $u \in \mathscr{U}[0, T]$ in controlled system, we obtain

$$
J_{f_{k}}\left(y_{k}\right) \longrightarrow J_{f}(y) .
$$

From the abovementioned equations, for all $u \in \mathscr{U}[0, T]$, the inequality

$$
J_{f}(\bar{y}) \leq J_{f}(y)
$$

holds. Namely, $\bar{y} \in S(f)$ and $S(f)$ is closed.

Since $S(f) \subset \mathscr{Y}_{S}[0, T]$ is closed and $\mathscr{Y}_{S}[0, T]$ is a compact set, we have the following theorem.

Theorem 5. Let (H1)-(H4) hold, and $S(f)$ is compact for each $f \in Y$.

Theorem 6. Let (H1)-(H4) hold, and then, $S$ is upper semicontinuous at each $f \in Y$.

Proof. Suppose $S$ is not upper semicontinuous; then, there exists open sets $O \subset C\left([0, T] ; R^{n}\right), \quad S(f) \subset O, \quad$ and $f_{k} \subset Y$ and $f_{k} \longrightarrow f$, such that some $\bar{y}_{f_{k}} \in S\left(f_{k}\right)$, but $\bar{y}_{f_{k}} \notin O$; in fact, by compactness, there exists a subsequence $\left\{\bar{y}_{f_{k_{1}}}\right\}$ of $\left\{\bar{y}_{f_{k}}\right\}$ such that $\bar{y}_{f_{k^{\prime}}} \longrightarrow \bar{y}$. Since $O$ is an open set and $\bar{y}_{f_{k}} \notin O$, then $\bar{y}_{f_{k^{\prime}}} \notin O$; we have that $\bar{y} \notin O$, but $\bar{y} \in S(f)$, and this contradicts $\bar{y} \in O$. We proved this theorem.

Hence, the set-valued mapping $S: Y \longmapsto 2^{Y_{S}[0, T]}$ is compact upper semicontinuous, from the Lemma 4.2 of [6], 
Definition 3.1 of [5], and Theorem 4.7 of [7], and therefore, we can obtain the theorem of stability.

Theorem 7. There exists a dense residual subset $W$ of $Y$, such that for any $f \in W, S(f)$ is stable in the sense of Hausdorff metric.

Remark 1. By Theorem 7, if $f \in Y$, the optimal control problem can be approximated in sense of Baire category.

Remark 2. We consider that the cost functional $J(y(\cdot), u(\cdot))=h(y(T))+\int_{0}^{T} f^{0}(t, y(t)) \mathrm{d} t$ contains $u(\cdot)$ implicitly. Namely, the cost functional is $J(y(\cdot), u(\cdot))=h(y(T))+\int_{0}^{T} f^{0}(t, y(t), u(t)) \mathrm{d} t$, and we need to let

$$
\begin{aligned}
\mathbf{f}(t, y(t), u(t)) & =\left(\begin{array}{c}
f(t, y(t), u(t)) \\
f^{0}(t, y(t), u(t))
\end{array}\right), \\
\mathbf{y}(t) & =\left(\begin{array}{c}
y(t) \\
y^{0}(t)
\end{array}\right), \\
\mathbf{y}_{0} & =\left(\begin{array}{c}
y_{0} \\
0
\end{array}\right) .
\end{aligned}
$$

Then, we can transform cost functional into $J(y(\cdot), u$ $(\cdot))=h(y(T))+y^{0}(T)$, and the discussion of stability is similar to the problem $(\mathrm{P})$.

\section{Example}

Consider the optimal problem

$$
\min _{u \in \mathscr{U}[0,1]} J(u)=(y(1))^{2}
$$

subject to the system

$$
\left\{\begin{array}{l}
\dot{y}(t)=f(t, y(t), u(t)), \quad t \in[0,1], \\
y(0)=0,
\end{array}\right.
$$

where $u \in \mathcal{U}[0,1]$ and

$$
\mathcal{U}[0,1]=\{u(t) \in[-1,1]\},
$$

where

$$
u(t)= \begin{cases}\alpha, & \text { if } t \in\left[0, \frac{1}{2}\right), \\ \beta, & \text { otherwise, }\end{cases}
$$

and $\alpha, \beta \in[-1,1]$. (H3).

One can easily show that $\mathcal{U}[0,1]$ satisfies hypothesis

(1) Let $f(t, y(t), u(t))=u(t)$, and $t \in[0,1]$. Then, for any $\alpha \in[-1,1]$,

$$
u^{\alpha}(t)= \begin{cases}\alpha, & \text { if } t \in\left[0, \frac{1}{2}\right), \\ -\alpha, & \text { otherwise. }\end{cases}
$$

We have $J\left(u^{\alpha}\right)=0$. That is,

$$
S(f)=\arg \min _{u \in \mathcal{U}[0,1]} J_{f}(u)=\left\{y^{\alpha}, \alpha \in[-1,1]\right\},
$$

where the optimal trajectory

$$
y^{\alpha}(t)= \begin{cases}\alpha t, & \text { if } t \in\left[0, \frac{1}{2}\right) \\ \alpha-\alpha t, & \text { otherwise. }\end{cases}
$$

(2) Let $f_{m}(t, y(t), u(t))=u(t)+(1 / m)$, and $t \in[0,1]$, for every $m=1,2, \ldots$; we have $J\left(u^{m}\right)=0$, where

$$
u^{m}(t)= \begin{cases}\alpha-\frac{1}{m}, & \text { if } t \in\left[0, \frac{1}{2}\right), \\ -\alpha-\frac{1}{m}, & \text { otherwise, }\end{cases}
$$

and $\alpha-(1 / m),-\alpha-(1 / m) \in[-1,1]$.

Then,

$$
S\left(f_{m}\right)=\arg \min _{u \in \mathcal{U}[0,1]} J_{f_{m}}(u)=\left\{y^{m}\right\}
$$

where the optimal trajectory

$$
y^{m}(t)= \begin{cases}\alpha t, & \text { if } t \in\left[0, \frac{1}{2}\right), \\ \alpha-\alpha t, & \text { otherwise. }\end{cases}
$$

Obviously, $\rho\left(f, f_{m}\right)=(1 / m) \rightarrow 0$ as $m \rightarrow+\infty$ and $y^{m} \rightarrow y^{\alpha}$.

\section{Conclusions}

In this paper, under the condition of the lack of good properties of optimal control, we discuss the compactness of the feasible trajectories set when the right-hand side function of the controlled system is disturbed and give a suitable metric for the space of the right-hand side function. Combined with the given assumptions, the space is a complete metric space. Then, according to the compact upper semicontinuous set-valued mapping, the continuous dependence of the optimal trajectory on the right-hand function and the stability of the cost functional on the righthand function are obtained in the sense of Baire category. 


\section{Data Availability}

No data were used to support this study.

\section{Conflicts of Interest}

The authors declare no conflicts of interest.

\section{Authors' Contributions}

Conceptualization, methodology, formal analysis, supervision, and original draft preparation were performed by Hongyong Deng. Validation, reviewing, and editing were carried out by Hongyong Deng, Wei Zhang, and Changchun Shen.

\section{Acknowledgments}

The authors thank Professor Wei Wei for a benefit discussion on this problem. This work was supported by the Science and Technology Program of Guizhou Province under grant no. [2016]1074; Fund Project of Guizhou University under grant no. YJRCXM[2018]019; and Science and Technology Foundation of Guizhou Province under grant nos. LKM[2013]21 and J[2015]2074.

\section{References}

[1] B. T. Kien, "Second-order optimality conditions and solution stability to optimal control problems governed by stationary Navier-Stokes equations," Acta Mathematica Vietnamica, vol. 44, no. 2, pp. 431-448, 2019.

[2] D. Rocha, C. J. Silva, and D. F. M. Torres, "Stability and optimal control of a delayed HIV model," Mathematical Methods in the Applied Sciences, vol. 41, no. 6, pp. 2251-2260, 2018.

[3] L. Bonifacius and K. Pieper, "Strong stability of linear parabolic time-optimal control problems," ESAIM: Control, Optimisation and Calculus of Variations, vol. 25, pp. 1-33, 2019.

[4] Y. Guo and T. Li, "Optimal control and stability analysis of an online game addiction model with two stages," Mathematical Methods in the Applied Sciences, vol. 2020, pp. 1-18, 2020.

[5] J. Yu, Z.-X. Liu, D.-T. Peng, D.-Y. Xu, and Y.-H. Zhou, "Existence and stability analysis of optimal control," Optimal Control Applications and Methods, vol. 35, no. 6, pp. 721-729, 2014.

[6] H. Y. Deng and W. Wei, "Existence and stability analysis for nonlinear optimal control problems with 1-mean equicontinuous controls," Journal of Industrial and Management Optimization, vol. 11, pp. 1049-1422, 2015.

[7] H. Y. Deng and W. Wei, "Stability analysis for optimal control problems governed by semilinear evolution equation," Advances in Difference Equations, vol. 2015, pp. 1-15, 2015.

[8] Y. Jiongmin and H. Lou, Concise Course of Optimal Control Theory, Higher Education Press, Beijing, China, 2006. 